\title{
Análise multivariada aplicada na identificação de fármacos antidepressivos. Parte II: Análise por componentes principais (PCA) e o método de classificação SIMCA
}

\author{
Janusa Goelzer Sabin', Marco Flôres Ferrão ${ }^{1,2,}{ }^{1}$, João Carlos Furtado ${ }^{2}$ \\ ${ }^{1}$ Grupo de Quimiometria - Departamento de Química e Física, ${ }^{2}$ Grupo de Sistemas e Processos Industriais - \\ Departamento de Informática, Universidade de Santa Cruz do Sul
}

Correspondência:

M. F. Ferrão

UNISC - Av. Independência 2293

96815-900, Santa Cruz do Sul, RS, Brasil

E-mail:ferrao@unisc.br
Neste trabalho a identificação e a discriminação de dois diferentes fármacos utilizados como antidepressivos foi estudada, empregando os espectros de reflexão difusa no infravermelho médio com transformada de Fourier (DRIFTS), juntamente com a análise de componentes principais (PCA) e o método de classificação SIMCA. Os espectros no infravermelho de amostras contendo diferentes concentrações dos princípios ativos cloridrato de amitriptilina e cloridrato de imipramina, foram coletados em um espectrofotometro NICOLET Magna 550, sendo realizadas 2 réplicas para cada amostra, com resolução de $4 \mathrm{~cm}^{-1}$ e 32 varreduras. A análise de componentes principais confirmou a existência de dois grupos distintos, correspondendo aos dois diferentes princípios ativos utilizados, além de evidenciar a presença de amostras anômalas no conjunto de dados que, possivelmente, iriam interferir na modelagem. Já o método de classificação SIMCA possibilitou o reconhecimento de amostras dos princípios ativos cloridrato de imipramina e cloridrato de amitriptilina com resultados indicando $100 \%$ de classificação correta das classes modeladas.

\section{INTRODUÇÃO}

O crescente consumo de fármacos antidepressivos faz com que o desenvolvimento de técnicas alternativas, mais eficazes e rápidas, sejam necessárias para o amplo controle de qualidade dos medicamentos industrializados. Em face disso, este trabalho propõe a implementação de um método de identificação de fármacos antidepressivos, a partir de dados do infravermelho, buscando discriminar as diferenças químicas entre as amostras estudadas.
Unitermos:

- Fármacos antidepressivos

- Reflexão difusa

- Infravermelho

- PCA

- SIMCA

- Quimiometria
Como ferramentas para o desenvolvimento desta metodologia é empregada a técnica de reflexão difusa no infravermelho com transformada de Fourier (DRIFT) associada ao método de análise PCA (Principal Component Analysis) e ao método de classificação SIMCA (Soft Independent Modeling by Class Analogy). A escolha da metodologia levou em consideração a redução do tempo de análise, a não destruição da amostra e a conseqüente não agressão ao meio ambiente por não gerar resíduos, além do baixo custo visto a demanda diária de análises. 


\section{Fármacos Antidepressivos Tricíclicos}

Entre os antidepressivos mais empregados encontramos aqueles da classe dos tricíclicos. Eles inibem a recaptação de neurotransmissores pela membrana pré-sináptica. Esta inibição da recaptação aumenta a concentração dos neurotransmissores em contato com os receptores, incrementando, assim, a atividade do circuito neural (Rang, Ritter, Dale, 2001; Silva, 2002).

Para este trabalho foram selecionados os princípios ativos amitriptilina e imipramina pertencentes à classe dos tricíclicos, que são agentes antidepressivos inibidores da recaptação da serotonina (5-HT). Ambas são aminas terciárias, sendo a amitriptilina derivada do dibenzocicloheptadieno e a imipramina derivada do iminodibenzil (Silva, 2002).

O princípio ativo imipramina também é denomidado por (dimetilamino-3-propil)-5-iminodibenzil (Silva, 2002).É um composto branco, cristalino, muito solúvel em água, apresentando fórmula estrutural conforme Figura 1.

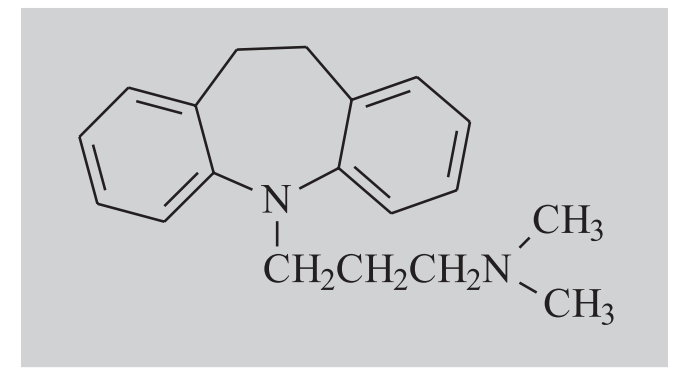

FIGURA 1 - Fórmula estrutural da imipramina.

Já a amitriptilina é também quimicamente denominada por (dimetilamino-3 propilideno)-5 dibenzo(a,d) cicloheptadieno (Silva, 2002). Trata-se de um composto branco, cristalino, prontamente solúvel em água, com massa molecular de 277,41 g/mol, apresentando fórmula estrutural conforme Figura 2.

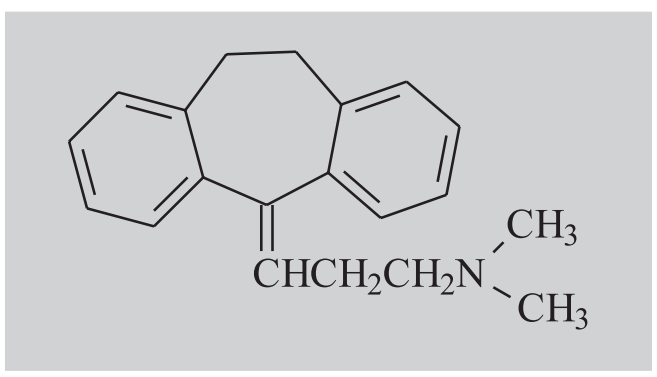

FIGURA 2 - Fórmula estrutural da amitriptilina.

\section{Espectroscopia por Reflexão Difusa no Infravermelho com Transformada de Fourier (DRIFTS)}

A reflexão difusa é principalmente observada em amostras sólidas pulverizadas, sendo sua aplicação prática muito difundida na literatura, podendo ser utilizada em equipamentos que operam na região do infravermelho próximo (Abdullah, Sherman, 1997; Birth, Hecht, 1987; Culler, 1993; Osborne, 1981; Osborne, Fearn, 1983; Osborne, Fearn, Hindle, 1993; Pasikatan et al., 2003; Stark, Luchter, 1986; Steuer, Schulz, 2003; van de Voort, 1992; Wilson, 1990), associada a sigla NIRS, Near Infrared Reflectance Spectroscopy, ou simplesmente NIRRS. No final dos anos 1970 e início dos 1980 foi demonstrada a utilidade deste fenômeno quando acessórios de reflexão difusa foram acoplados com espectrômetros interferométricos com transformada de Fourier, sendo conhecida a técnica resultante pela sigla DRIFTS, Diffuse Reflection Infrared Fourier Transform Spectroscopy (Fuller, Griffiths, 1978; van de Voort, Ismail, 1991; Reeves III, Zapf, 1998; Wilson, Tapp, 1999).

A reflexão difusa é observada quando uma luz incide em matriz descontínua, penetra na amostra (tipo amostra em pó, papel) e reflete contendo informações espectrais. O caminho percorrido pela luz no interior da matriz pode ser considerado aleatório devido a múltiplas reflexões, algumas das quais após percorrer o interior de algumas partículas que constituem a amostra, conforme ilustrado pela Figura 3 (Wetzel, 1983). Desta forma, a luz refletida pode ser atenuada por absorção e o espectro resultante é similar ao obtido através da técnica no infravermelho por transmissão utilizando KBr. Uma importante diferença entre a transmissão e a reflexão é devida ao diferente caminho óptico percorrido pela luz. Enquanto na transmissão o caminho óptico é constante para todo número de onda, na reflexão o caminho pode ser variável. É bem sabido que em regiões do espectro em que a amostra absorve fracamente a luz penetra mais profundamente na matriz, enquanto o contrário acontece onde há forte absorção. Portanto, ao se comparar o espectro obtido por transmissão (pastilha de $\mathrm{KBr}$ ) com o obtido por reflexão, as intensidades relativas das bandas serão diferentes. Por exemplo, as bandas fracas no espectro por transmissão aparecerão mais fortes na reflexão.

É importante salientar que num experimento de reflexão difusa será também observada a reflexão especular, sendo mais importante aquela que ocorre na interface ar/superfície da matriz, mas também ocorrendo nas faces das partículas, mas cujo efeito pode ser minimizado pela redução do tamanho da partícula. A reflexão especular é de maior intensidade na região em que a amostra apresenta forte absorção e neste caso podem ocorrer graves distor- 
ções no espectro obtido (Ferrão, 2001). Deve-se ressaltar a diferença marcante entre infravermelho médio e próximo. No infravermelho próximo as distorções nos espectros são quase imperceptíveis, enquanto que no infravermelho médio as distorções são mais freqüentes. Uma maneira de contornar esse efeito não desejado é diluindo a amostra em matriz não absorvente, como KBr (Olinger, Griffiths, 1983a; Olinger, Griffiths, 1983b).

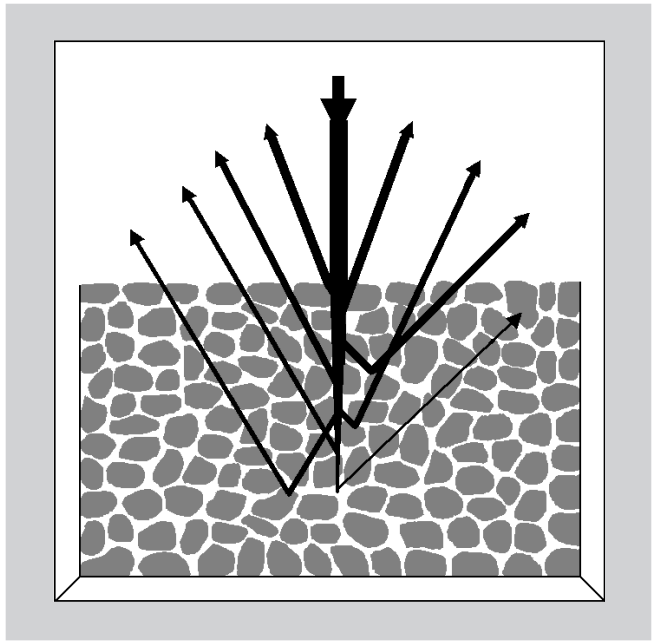

FIGURA 3 - Reflexão especular e difusa de uma onda eletromagnética em uma amostra (Wetzel, 1983).

Um maior detalhamento desta técnica, bem como de outras técnicas de reflexão empregadas em análises que potencialmente aplicam informações químicas obtidas via espectroscopia no infravermelho com transformada de Fourier, pode ser encontrado em recente revisão publicada (Ferrão, 2001).

\section{Espectroscopia no Infravermelho Aplicada na Análise de Medicamentos}

A reflexão no infravermelho vem sendo utilizada na análise de medicamentos desde o início dos anos 1970, porém sua expansão veio a ocorrer nos anos 1990 (Corti, Dreassi, Leonardi, 1993), principalmente com a evolução da informatização dos equipamentos e da associação destes com as ferramentas de análise mulivariada.

Um dos focos dos trabalhos realizados vem sendo a aplicação da espectroscopia no infravermelho próximo para o controle de medicamentos nas diversas fases de produção (Plugge, van der Vlies,1992; MacDonald, Prebble, 1993; Dreassi et al., 1996; Blanco et al., 1998; Blanco et al., 1999a; Blanco et al., 2000a; Herkert, Prinz, Kovar, 2001). Porém a maior parte da literatura disponível trata de aplicações da espectroscopia por reflexão difusa no infraver- melho próximo na quantificação de ingredientes ativos, sendo a maior parte dos trabalhos aplicada ao produto final (Trafford et al., 1999; Blanco et al., 1999b; Blanco et al., 1999c; Guo et al., 1999; Blanco et al., 2000b; Blanco et al., 2001; Laasonen et al., 2003; Sun, Xiang, An, 2004).

Também, ao longo deste últimos anos vêm sendo realizados trabalhos que comparam o desempenho da espectroscopia no infravermelho próximo por transmissão com outras técnicas espectroscópicas como infravermelho próximo por reflexão difusa (Gottfries et al., 1996) e espectroscopia na região do ultravioleta (Eustaquio et al., 1998; Thosar et al., 2001). Raros são os trabalhos que empregam a espectroscopia por reflexão difusa no infravermelho com transformada de Fourier (DRIFTS), a exemplo do trabalho publicado por Yang e Irudayaraj, em 2002, no qual se comparam a espectroscopia FT-NIR com DRIFTS para a determinação de vitamina C. Neste trabalho os pesquisadores também utilizam outras técnicas como espectroscopia por reflexão total atenuada no infravermelho com transformada de Fourier (FTIR-ATR), espectroscopia fotoacústica no infravermelho com transformada de Fourier (FTIR-PAS) e espectroscopia Raman com transformada de Fourier (FT-Raman). Já a avaliação da degradação de princípios ativos empregando espectroscopia no infravermelho foi pouco estudada (Drennen, Lodder, 1990).

\section{Análise Multivariada}

Os métodos de análise multivariada são assim chamados pois, em caso do emprego das técnicas espectroscópicas no infravermelho, é possível manipular dados de absorbância espectral associados a mais de uma freqüência ao mesmo tempo. Estes métodos têm recentemente tornado possível modelar propriedades químicas e físicas de sistemas simples e complexos a partir de seus dados espectroscópicos. As análises qualitativas e quantitativas de medicamentos, utilizando espectroscopia na região do infravermelho, expandiram-se a partir do momento em que os dados gerados por um espectrofotômetro FT-IR puderam ser digitalizados, habilitando os métodos estatísticos na resolução de problemas da análise química. A possibilidade de utilizar várias freqüências do espectro tem aumentado o tipo de amostras que podem ser analisadas por espectroscopia no infravermelho (Pasquini, 2003; Sabin, Banet, Ferrão, 2003; Yoon et al., 2004).

\section{Análise por Componentes Principais (PCA)}

A análise por componentes principais (PCA) é um dos métodos mais comuns empregados na análise de informações (Brown, 1995; Ferreira, 2002), sendo principal- 
mente utilizada pela sua capacidade de compressão dos dados em função da existência de correlação entre diversas variáveis medidas. Quando aplicamos um algoritmo de PCA num conjunto de variáveis, como por exemplo, espectros no infravermelho, o conjunto original destas variáveis é substituído por um novo conjunto de variáveis denominado de Componentes Principais (CPs). A principal característica deste novo conjunto é a ortogonalidade, porém o mesmo é facilmente reconstruído a partir da combinação linear das variáveis originais (espectros). Como vantagem, o novo conjunto de variáveis (CPs), geralmente concentra a maior parte da informação (variância) em poucas variáveis, diminuindo assim a dimensionalidade dos dados, sem perda significativa da informação química. A maioria dos aplicativos disponíveis utilizam a técnica de decomposição do valor singular (SDV) para obter as CPs, sendo neste caso a primeira componente principal (CP1) definida na direção (eixo) de maior variância do conjunto de variáveis originais. De forma decrescente em termos de variação são definidas as demais componentes principais, porém estas serão sempre ortogonais a CP1 e entre si. Por exemplo, um sistema que seja reduzido a $3 \mathrm{CPs}(\mathrm{CP} 1, \mathrm{CP} 2 \mathrm{e}$ CP3) se assemelha ao sistema cartesiano de coordenadas, em que todos os eixos são linearmente independentes, isto é, ortogonais entre si. Para os casos de conjuntos de espectros consideramos inicialmente matriz de dados X (m $\mathrm{x} \mathrm{n}$ ), sendo que $\mathrm{m}$ corresponde ao número de amostras (espectros) e n o número de variáveis (freqüências do espectro), que pode ser decomposta em 3 outras matrizes, U, S e V (Ferreira, 2002), conforme expressão 1:

$$
\mathrm{X}=\mathrm{USV}^{\mathrm{t}}
$$

As colunas de U e V são ortogonais. A matriz V é a matriz dos pesos, em que a primeira coluna contém os pesos de $\mathrm{PC} 1$ e assim por diante. O produto $\mathrm{U} \times \mathrm{S}$ corresponde à matriz $\mathrm{T}$ dos escores. Por fim, $\mathrm{S}$ é matriz diagonal, cujos elementos (valores singulares) contêm informações sobre a quantidade de variância que cada componente principal descreve. A matriz S é importante na determinação da dimensionalidade intrínseca da matriz de dados, podendo os analistas definir quantas CPs ou fatores devem ser utilizados para análises posteriores. Os autovalores que forem pequenos serão excluídos e as informações relevantes podem, de alguma maneira, ser separadas, eliminando-se, assim, os ruídos experimentais.

\section{Métodos de Reconhecimento de Padrões}

Dentre os métodos de reconhecimento de padrões baseados na similaridade encontramos o KNN (k-nearest neighbor) e o SIMCA (soft independent modeling by class analogy). A classificação KNN utiliza-se da comparação entre as distâncias das amostras, sendo esta obtida pela distância Euclidiana calculada para cada par de amostras (Kowalski, Bender, 1972), conforme expressão 2.

$$
d_{a b}=\left[\sum_{j}^{m}\left(x_{a j}-x_{b j}\right)^{2}\right]^{1 / 2}
$$

A classificação de amostras externas às amostras modeladas (utilizadas na construção de classes), é determinada através do cálculo da distância multivariada destas novas amostras com relação as k-ésimas amostras vizinhas empregadas na modelagem. O método mais comum para a validação deste tipo de classificação baseiase na validação cruzada para a qual cada uma das amostras do conjunto de modelagem é retirada uma de cada vez. Com isto, são determinados quantos vizinhos k-ésimos devem ser considerados para posterior classificação. Finalmente, para estimar a classe de uma amostra externa são verificadas quantas vezes amostras de uma mesma classe são atribuídas como vizinhas à amostra em questão, sendo considerada a classe final aquela à qual maior número de vizinhos seja relacionado a esta amostra.

Por outro lado, o método SIMCA (Sjöström, Kowalski, 1979; Wold et al., 1983) é construído a partir de modelos baseados em componentes principais, no qual cada classe corresponde a um conjunto de treinamento. Um número ótimo de componentes principais é determinado independentemente para cada classe e o modelo final é obtido através da definição da fronteira entre cada PCA modelado. Como conseqüência, uma hipercaixa com tantas dimensões quantas forem o número de CPs mais adequados para cada classe é obtida. Uma vez definidas as hipercaixas é importante verificar se existem classes com sobreposição. O poder de discriminação dos modelos SIMCA se baseia na não existência de sobreposição entre duas classes diferentes, bem como na maior distância interclasses possível. A classificação de uma amostra externa àquelas empregadas na modelagem SIMCA é baseada nas diferentes projeções possíveis no espaço dos escores avaliando-se se a mesma encontra-se dentro das fronteiras definidas para a respectiva classe em todas as projeções obtidas.

\section{MATERIAIS E MÉTODOS}

\section{Aquisição dos Espectros}

Espectros no infravermelho médio foram coletados em um espectrofotômetro FT-IR Magna 550 da Nicolet, 
sendo realizadas 2 réplicas para cada amostra, com resolução de $4 \mathrm{~cm}^{-1}, 32$ varreduras para cada espectro e a região utilizada foi de $650-4000 \mathrm{~cm}^{-1}$. O instrumento foi equipado com um acessório de reflectância difusa, e os sinais expressos em $\log (1 / \mathrm{R})$. Foram utilizadas 18 amostras no total, 9 contendo o princípio ativo cloridrato de imipramina (18 espectros) e 9 contendo o princípio ativo cloridrato de amitriptilina (18 espectros), com diferentes concentrações compreendidas entre 6 e $40 \%$ do princípio ativo em amido.
Os conjuntos de espectros das amostras de cloridrato de imipramina e cloridrato de amitriptilina, em que foi utilizado o princípio ativo proveniente do fornecedor Cristália Produtos Químicos Farmacêuticos Ltda, encontram-se nas Figuras 4 e 5.

\section{Modelagem PCA}

Os modelos foram desenvolvidos através do aplicativo Pirouette 2.7 da Infometrix empregando a aná-

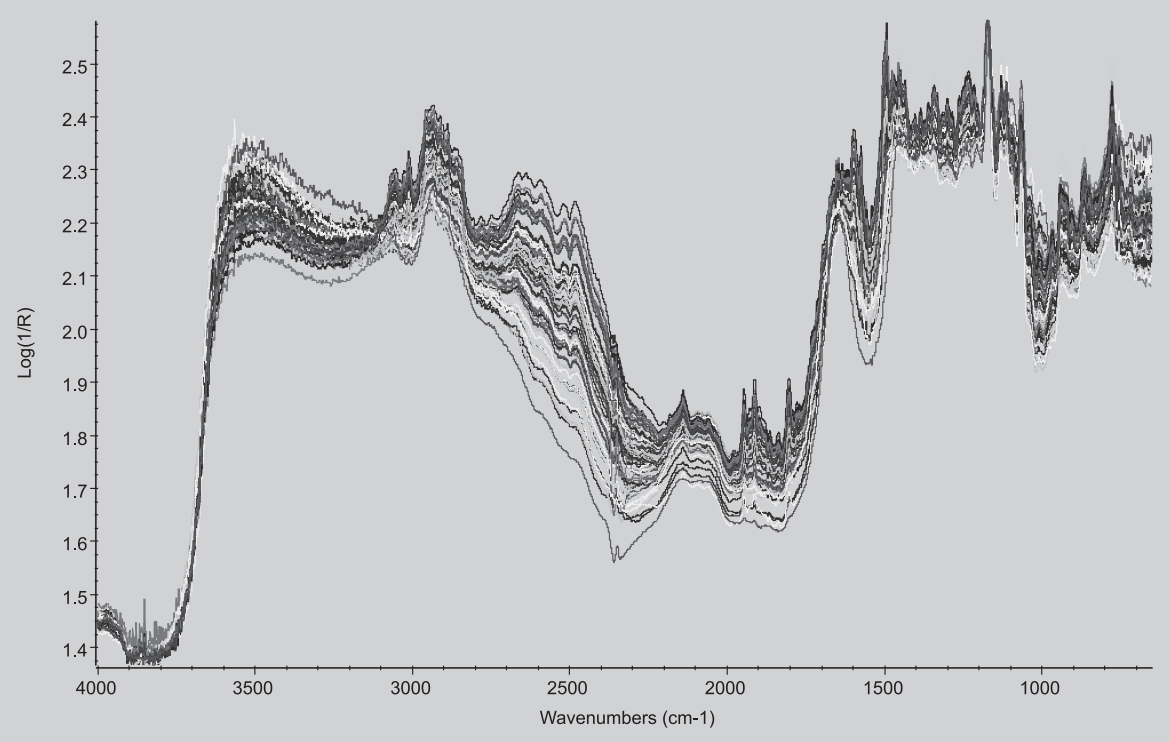

FIGURA 4 - Conjunto de espectros do princípio ativo cloridrato de imipramina.

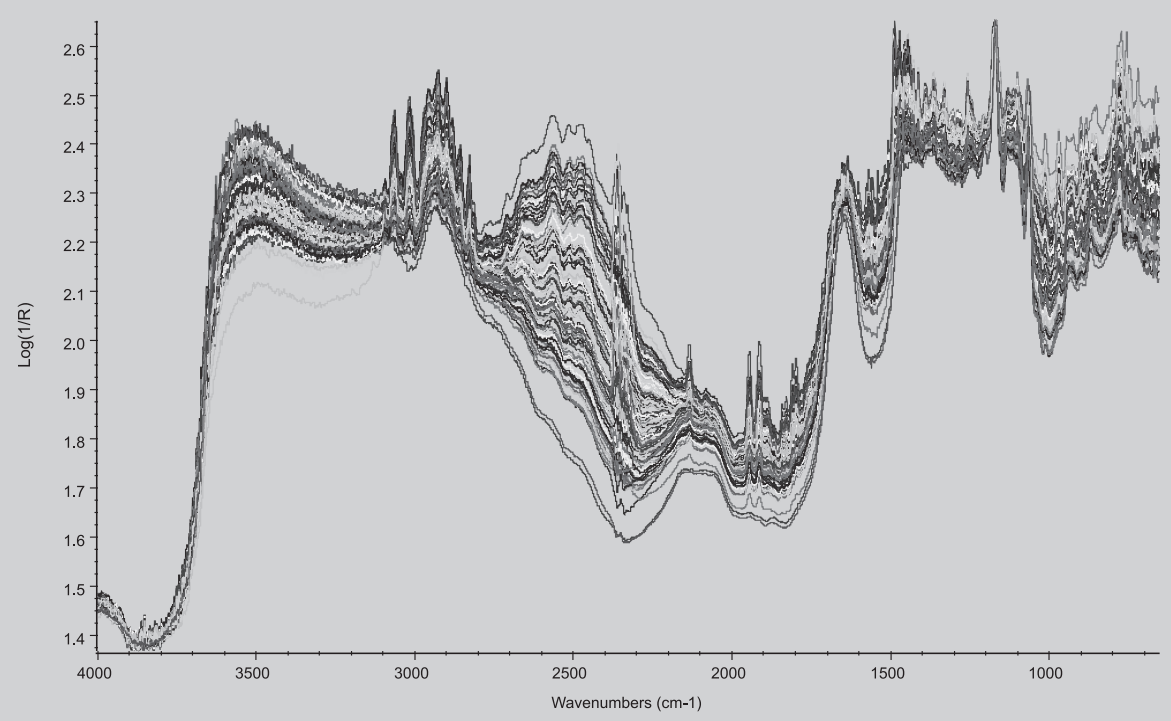

FIGURA 5 - Conjunto de espectros do princípio ativo cloridrato de amitriptilina. 
lise de componentes principais (PCA). Previamente foi selecionado o conjunto de regiões para modelagem com maior número de informações de interesse, ou seja, o conjunto que melhor correlacionava os espectros e qualificação dos princípios ativos. As regiões selecionadas foram de 800 a $1799 \mathrm{~cm}^{-1}$ e de 2800 a $3498 \mathrm{~cm}^{-1}$.

A modelagem foi realizada utilizando-se 36 amostras no total 18 amostras de cloridrato de imipramina (1 a 18) e 18 amostras de cloridrato de amitriptilina (19 a 36). O pré-processamento usado foi o auto-escalamento que consiste em centrar os dados na média e dividi-los cada um pelo desvio-padrão, de forma que todas as variáveis passam a ter a mesma variância.

À matriz de dados, foi empregado ainda a técnica de tratamento de correção de espalhamento de luz (MSC), com a finalidade de corrigir o efeito do espalhamento de luz presente nos espectros obtidos por técnicas de reflexão, causados, principalmente, pela falta de homogeneidade ótica das amostras.

\section{Modelagem SIMCA}

Para o método de classificação SIMCA, os modelos também foram desenvolvidos através do aplicativo Pirouette 2.7 da Infometrix. A modelagem SIMCA foi realizada com 10 amostras (20 espectros), correspondendo aos princípios ativos cloridrato de imipramina e cloridrato de amitriptilina. Para a validação foram empregadas 8 amostras (16 espectros), sendo 4 amostras de cloridrato de imipramina e 4 amostras de cloridrato de amitriptilina. Foram construídos diversos modelos fazendo-se variar o pré-processamento dos dados e/ou o emprego da correção do sinal multiplicativo, avaliando-se a habilidade de cada modelo em classificar corretamente as amostras de validação.

\section{RESULTADOS E DISCUSSÃO}

$\mathrm{Na}$ análise por componentes principais (PCA) um pré-processamento nos dados foi necessário para atribuir pesos equivalentes aos dados espectrais das amostras.

A PCA mostrou que com seis componentes principais é possível descrever $90,22 \%$ dos dados, sendo $53,17 \%$ da variância total descrita pela primeira componente principal.

O gráfico da Figura 6 permite verificar a necessidade de remover algumas amostras do conjunto de dados, uma vez que apresentam diferenças das demais contidas no conjunto podendo, portanto, influenciar o modelo de forma negativa. Entretanto, podemos observar que nenhuma das amostras apresentou alto resíduo, não sendo por isso necessária a remoção de amostra modelada.

A análise do gráfico de escores, Figura 7, mostra a separação das amostras em dois grandes grupos, ou seja, as amostras contendo o princípio ativo cloridrato de imipramina (amostras numeradas de 1 a 18) são discriminadas daquelas contendo o princípio ativo cloridrato de amitriptilina (amostras numeradas de 19 a 36).

Já para a modelagem SIMCA o melhor modelo

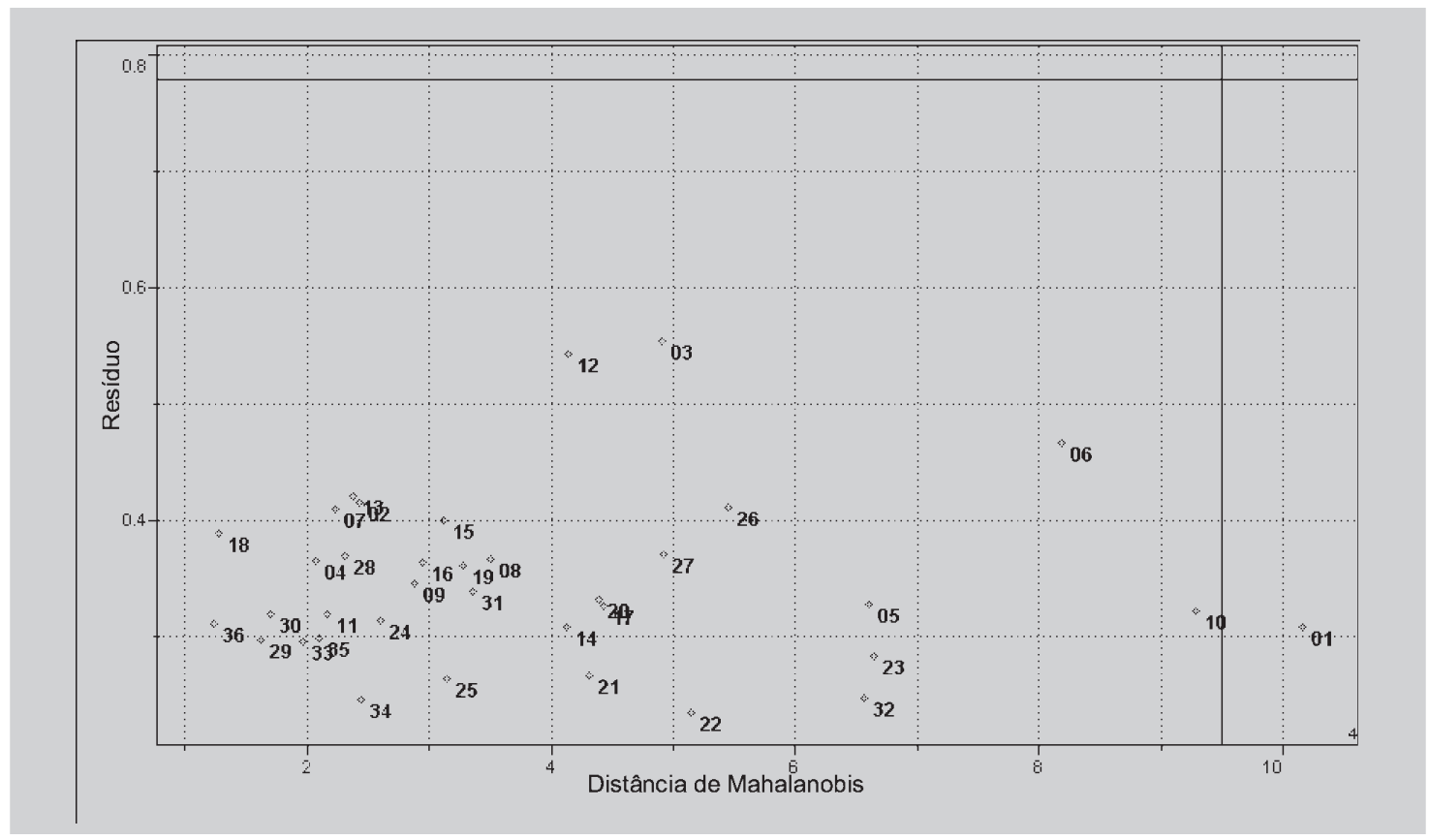

FIGURA 6 - Gráfico do diagnóstico de amostras anômalas (outlier diagnostics) para o PCA com os dados autoescalados e MSC. 


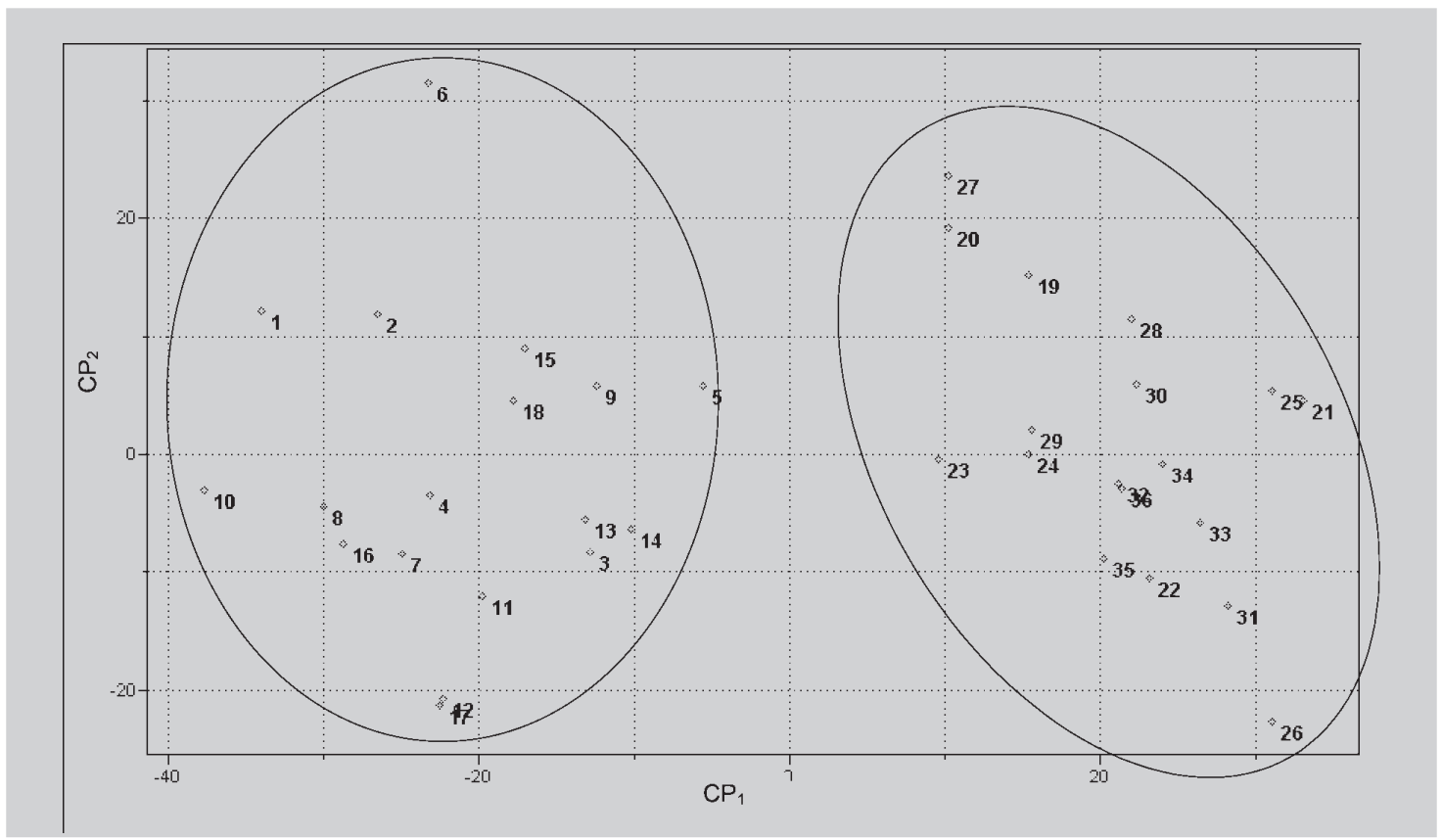

FIGURA 7 - Gráfico $\mathrm{CP}_{1} \mathrm{xCP}_{2}$ dos escores para os espectros das amostras de cloridrato de imipramina e cloridrato de amitriptilina, do PCA com os dados auto-escalados e MSC.

empregou os dados auto-escalados e 4 componentes principais para ambos os princípio ativos (cloridrato de imipramina e cloridrato de amitriptilina) que acumulam $91,21 \%$ e $93,21 \%$ das informações, respectivamente. À matriz de dados foi empregado ainda, a técnica de tratamento de correção de espalhamento de luz (MSC).

Das 8 amostras empregadas na validação (16 espectros), 4 foram classificadas como amostras de cloridrato de imipramina (classe 1) e 4 como amostras de cloridrato de amitriptilina (classe 2), conforme Figura 8. Estes resultados indicam $100 \%$ de classificação correta das respectivas classes modeladas.

\section{CONCLUSÕES}

A identificação e discriminação de medicamentos é importante para garantir a qualidade e autenticidade dos mesmos. Neste sentido a análise exploratória em conjunto com a espectroscopia por reflexão difusa no infravermelho com transformada de Fourier (DRIFTS) mostraram-se viáveis, por possibilitarem a análise direta, sem tratamento prévio das amostras, o que reduz consideravelmente o tempo dispensado às análises químicas.

O emprego da análise por componentes principais (PCA) não evidenciou existência de amostras anômalas no conjunto, as quais poderiam influenciar negativamente nos resultados, enquanto que a técnica SIMCA possibilitou a classificação correta das amostras contendo os diferentes

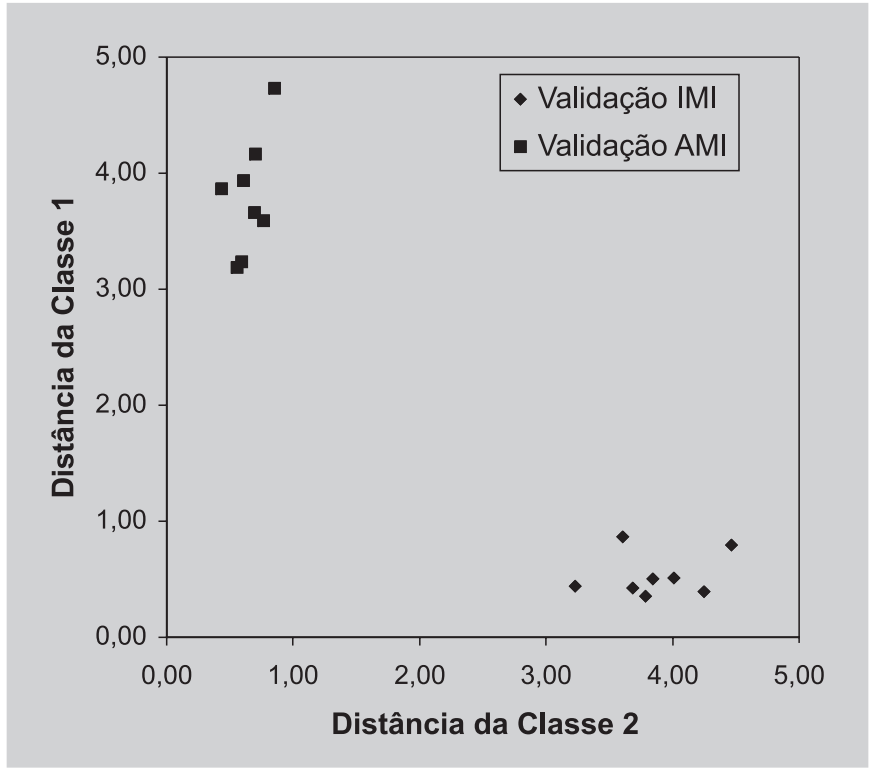

FIGURA 8 - Gráfico das distâncias entre as classes modeladas para o SIMCA com quatro componentes principais.

fármacos antidepressivos, importante para o controle de qualidade dos produtos, porém dificilmente visualizados diretamente na matriz de dados (conjunto de espectros).

Ainda pode-se afirmar que esta metodologia pode ser utilizada para outros medicamentos, uma vez que neste trabalho ficou demonstrado que mesmo compostos com 
ação farmacológica e estrutura química muito semelhantes podem ser facilmente diferenciados e identificados.

\section{AGRADECIMENTOS}

Os autores agradecem à empresa Cristália Produtos Químicos Farmacêuticos Ltda, pela doação das amostras dos fármacos, à Central Analítica da UNISC, pela utilização dos equipamentos, e à Fundação de Amparo à Pesquisa do Estado do Rio Grande do Sul, pelo apoio financeiro concedido.

\section{ABSTRACT \\ Multivariate analysis to applied in the identification of antidepressants. Part II: principal components analysis (PCA) and soft independent modeling of class analogies (SIMCA)}

In this work the certification of two different drugs used as antidepressants was studied, using diffuse reflectance infrared Fourier transform spectroscopy (DRIFTS), together with the analysis of principal components (PCA) and the method of soft independent modeling of class analogies (SIMCA). The DRIFT spectra of samples with different concentrations of the active principles amitriptiline and imipramine hydrochlorides had been collected in Magna 550 spectrofotometer, two spectra for each sample, with resolution of $4 \mathrm{~cm}^{-1}$ and 32 scans. The PCA confirmed the existence of two distinct groups, corresponding to the two different active principles used. Otherwise the method of classification SIMCA made possible the recognition of samples of the principles amitriptyline and imipramine hydrochlorides with results indicating $100 \%$ of correct classification.

UNITERMS: Antidepressants. Difuse Reflection. Infrared. PCA. SIMCA. Chemometrics.

\section{REFERÊNCIAS BIBLIOGRÁFICAS}

ABDULLAH, A.H., SHERMAN, W.F. Kramers-Kroning type analysis of short spectral range reflection spectra. Vib. Spec., Amsterdam, v.13, n.2, p.133-142, 1997.

BIRTH, G.S., HECHT, H.G. The physics of near-infrared reflectance. In: WILIAMS,P.; NORRIS,K., eds. Nearinfrared technology in the agricultural and food industries. St. Paul: American Association of Cereal Chemists, 1987. p.1-15.
BLANCO M., COELlO J., EUSTAQUIO, A., ITURRIAGA H., MASPOCH S. Analytical control of pharmaceutical production steps by near infrared reflectance spectroscopy. Anal. Chim. Acta, Amsterdam, v. 392, n. 2-3, p.237-246, 1999a.

BLANCO M., COELlO J., EUSTAQUiO A., ITTURRIAGA H., MASPOCH S. Development and validation of methods for the determination of miokamycin in various pharmaceutical preparations by use of near infrared reflectance spectroscopy. Analyst, London, v. 124, n. 7, p. 1089-1092, 1999 b.

BLANCO M., COELLO J., EUSTAQUIO A., ITURRIAGA H., MASPOCH S. Development and validation of a method for the analysis of a pharmaceutical preparation by near-infrared diffuse reflectance spectroscopy. J. Pharm. Sci., Washington, v. 88, n.5, p.551-556, 1999c.

BLANCO M., COELLO J., ITURRIAGA H., MASPOCH S., SERRANO D. Near-infrared analytical control of pharmaceuticals. A single calibration model from mixed phase to coated tablets. Analyst, London, v. 123, n. 11, p. 2307-2312, 1998.

BLANCO M., COELLO J., ITURRIAGA H., MASPOCH S., POU N. Influence of the procedure used to prepare the calibration sample set on the performance of near infrared spectroscopy in quantitative pharmaceutical analyses. Analyst, London, v. 126, n. 7, p.1129-1134, 2001.

BLANCO M., COELLO J., ITURRIAGA H., MASPOCH S., POU N. Development and validation of a near infrared method for the analytical control of a pharmaceutical preparation in three steps of the manufacturing process. Fresenius J. Anal. Chem., Berlin ,v. 368 , n. 5, p. 534-539, 2000a.

BLANCO M., EUSTAQUIO A., GONZALEZ J.M., SERRANO D. Identification and quantitation assays for intact tablets of two related pharmaceutical preparations by reflectance near-infrared spectroscopy: validation of the procedure. J. Pharm. Biomed. Anal., Amsterdam, v.22, n.1, p.139-148, 2000b.

BROWN, S.D. Chemical systems under indirect observation: Latent properties and chemometrics. Appl. Spectrosc., Baltimore, v.49, n.12, p.14A-31A, 1995. 
CORTI P., DREASSI E., LEONARDI S. Near infrared reflectance analysis: features and applications in pharmaceutical and biomedical analysis. Farmaco, Pavia, v.48, n.2, p.3-20, 1993.

CULLER, S.R. Diffuse reflectance infrared spectroscopy: Sampling techniques for qualitative / quantitative analysis of solids. In: COLEMAN, P.B. Practical sampling techniques for infrared analysis. Boca Raton: CRC Press, 1993. cap.4, p.93-105.

DREASSI E., CERAMELLI G., CORTI P., PERRUCCIO P.L., LONARDI S. Application of near-infrared reflectance spectrometry to the analytical control of pharmaceuticals: ranitidine hydrochloride tablet production. Analyst, London, v. 121, n. 2, p.219-222, 1996.

DRENNEN J.K., LODDER R.A. Nondestructive nearinfrared analysis of intact tablets for determination of degradation products. J. Pharm. Sci., Washington, v.79, n.7, p.622-627, 1990.

EUSTAQUIO A., GRAHAM P., JEE R.D., MOFFATT A.C., TRAFFORD A.D. Quantification of paracetamol in intact tablets using near-infrared transmittance spectroscopy. Analyst, London, v.123, n.11, p.2303-2306, 1998.

FERRÃO, M.F. Técnicas de reflexão no infravermelho aplicadas na análise de alimentos. Tecno-lóg., Santa Cruz do Sul, v.5, n.1, p.63-85, 2001.

FERREIRA, M.M.C. Multivariate QSAR. J. Braz. Chem. Soc., São Paulo, v.13, n.6, p.742-753, 2002.

FULLER, M.P.E., GRIFFITHS, P.R. Diffuse reflectance measurements by infrared Fourier transform spectrometry. Anal. Chem., Oxford, v.50, p.1906-1910, 1978.

GOTTFRIES J., DEPUI H., FRANSSON M., JONGENEELEN M., JOSEFSON M., LANGKILDE F.W., WITTE D.T. Vibrational spectrometry for the assessment of active substance in metoprolol tablets: a comparison between transmission and diffuse reflectance near-infrared spectrometry. J. Pharm. Biomed. Anal., Amsterdam, v.14, n.11, p.1495-1503, 1996.

GUO J.H., SKINNER G.W., HARCUM W.W., MALONE J.P., WEYER L.G. Application of near-infrared spectroscopy in the pharmaceutical solid dosage form. Drug Dev. Ind. Pharm., New York, v.25, n.12, p.1267$1270,1999$.
HERKERT T., PRINZ H., KOVAR K. One hundred percent online identity check of pharmaceutical products by near-infrared spectroscopy on the packaging line. Eur. J. Pharm. Biopharm., London, v. 51, n.1, p.9-16, 2001.

KOWALSKI, B.R., BENDER, C.F. Pattern recognition. A powerful approach to interpreting chemical data. $J$. Am. Chem. Soc., Washington, v. 94, p. 5632-5639, 1972.

LAASONEN M., HARMIA-PULKKINEN T., SIMARD C., RASANEN M., VUORELA H. Development and validation of a near-infrared method for the quantitation of caffeine in intact single tablets. Anal. Chem., Washington, v.75, n.4, p.754-760, 2003.

LIMA, D.R. Manual de farmacologia clínica, terapêutica e toxicologia. Rio De Janeiro: Guanabara Koogan, 1995. $1252 \mathrm{p}$.

MACDONALD B.F., PREBBLE K.A. Some applications of near-infrared reflectance analysis in the pharmaceutical industry. J Pharm Biomed Anal., Amsterdam, v. 11, n.11-12, p.1077-1085, 1993.

OLINGER, J.M.E., GRIFFITHS, P.R. Effects of sample dilution and particle size/morphology on diffuse reflection spectra of carbohydrate systems in the near- and midinfrared. Part I: Single analytes. Appl. Spectrosc., Baltimore, v.47, p.687-694, 1993a.

OLINGER, J.M.E., GRIFFITHS, P.R. Effects of sample dilution and particle size/morphology on diffuse reflection spectra of carbohydrate systems in the near- and midinfrared. Part II: Durum wheat. Appl. Spectrosc., Baltimore, v. 47, p.695-701, 1993b.

OSBORNE, B.G. Principles and practice of near-infrared (NIR) reflectance analysis. J. Food Technol., Oxford, v.16, p.13-19, 1981.

OSBORNE, B.G., FEARN, T. Collaborative evaluation of near-infrared reflectance analysis for the determination of protein, moisture and hardness in wheat. J. Sci. Food Agric., London, v.34, p.1011-1017, 1983.

OSBORNE, B.G., FEARN,T., HINDLE, P.H. Pratical NIR spectroscopy: With applications in food and beverage analysis. Harlow: Longman Scientific \& Technical, 1993. 227 . 
PASIKATAN, M.C., HAQUE,E., SPILLMAN, C.K., STEELE, J.L., MILLIKEN, G.A. Granulation sensing of first-break ground wheat using a near-infrared reflectance spectrometer: studies with soft red winter wheats. J. Sci. Food Agric., London, v.83, p.151-157, 2003.

PASQUINI, C. Near infrared spectroscopy: fundamentals practical aspects and analytical applications. J. Braz. Chem. Soc., São Paulo, v.14, n.2, p.138-219, 2003.

PLUGGE W., VAN DER VLIES C. The use of near infrared spectroscopy in the quality control laboratory of the pharmaceutical industry. J Pharm Biomed Anal., Amsterdam, v. 10, n. 10-12, p.797-803, 1992.

RANG, H.P., RITTER, J.M., DALE, M.M. Farmacologia. 4. ed. Rio de Janeiro: Guanabara Koogan, 2001. 703p.

REEVES III, J.B., ZAPF, C.M. Mid-infrared diffuse reflectance spectroscopy for discriminant analysis of food ingredients. J. Agric. Food Chem., Easton, v.46, p.36143622, 1998.

SABIN, J.G., BANET, E.V., FERRÃO, M.F. Análise exploratória aplicada na identificação de fármacos antidepressivos. Parte I: Análise hierárquica de agrupamentos. Tecno-lóg., Santa Cruz do Sul, v.7, n.1, p. 69-88, 2003.

SILVA, P. Farmacologia. 5.ed. Rio de Janeiro: Guanabara Koogan, 2002. 1374p.

SJÖSTRÖM, M., KOWALSKI, B.R. A comparison of five pattern recognition methods based on the classification results from six real data bases. Anal. Chim. Acta, Amsterdam, v.112, p.11-30, 1979.

STARK, E.E., LUCHTER, K. Near-infrared analysis (NIRA): A technology for quantitative and qualitative analysis. Appl. Spectrosc. Rev., New York, v.22, p.335-339, 1986.

STEUER, B., SCHULZ, H. Near-infrared analysis of fennel (Foeniculum vulgare Miller) on different spectrometers - Basic considerations for a reliable network. Phytochem. Anal., Sussex, v.14, p.285-289, 2003.

SUN M.L., XIANG B.R., AN D.K. A near-infrared diffuse reflectance analysis method for the noninvasive quantitative analysis of ambroxol hydrochloride tablets. Yao Xue Xue Bao, Peking, v.39, n.1, p.60-63, 2004.
THOSAR S.S., FORBESS R.A., EBUBE N.K., CHEN Y., RUBINOVITZ R.L., KEMPER M.S., REIER G.E., WHEATLEY T.A., SHUKLA A.J. A comparison of reflectance and transmittance near-infrared spectroscopic techniques in determining drug content in intact tablets. Pharm. Dev. Technol., New York, v.6, n.1, p.19-29, 2001.

TRAFFORD A.D., JEE R.D., MOFFAT A.C., GRAHAM P. A rapid quantitative assay of intact paracetamol tablets by reflectance near-infrared spectroscopy. Analyst, London, v. 124, n. 2, p.163-167, 1999.

VAN DE VOORT, F.R. Fourier transform infrared spectroscopy applied to food analysis. Food Res. Int., Ottawa, v.25, n.5, p.397-403, 1992.

VAN DE VOORT, F.R., ISMAIL, A.A. Proximate analysis of foods by mid-FTIR spectroscopy. Trends Food Sci. Technol., v.2, p.13-17, 1991.

WETZEL, D.L. Near-infrared reflectance analysis sleeper among spectroscopic techniques. Anal. Chem., Oxford, v.55, n.12, p.1165A-1176A, 1983.

WILSON, R.H. Fourier transform mid-infrared spectroscopy for food analysis. Trends Anal. Chem., Amsterdam, v.9, p.127-131, 1990.

WILSON, R.H., TAPP, H.S. Mid-infrared spectroscopy for food analysis: recent new applications and relevant developments in sample presentation methods. Trends Anal. Chem., Amsterdam, v.18, p.85-93, 1999.

WOLD, S., ALBANO, C., DUNN, W.J., ESBENSEN, K., HELLBERG, S., JOHANSSON, E., SJÖSTRÖM, M. Pattern recognition: Finding and using regularities in multivariate data. In: MARTENS, H., RUSSWURM, H. Food Research and Data Analysis, eds. Essex: Applied Science, 1983. p.147-188.

YANG H., IRUDAYARAJ J. Rapid determination of vitamin C by NIR, MIR and FT-Raman techniques. J. Pharm. Pharmacol., London, v.54, n.9, p.1247-1255, 2002.

YOON W.L., JEE R.D., CHARVILL A., LEE G., MOFFAT A.C. Application of near-infrared spectroscopy to the determination of the sites of manufacture of proprietary products. J. Pharm. Biomed. Anal., Amsterdam, v.34, n.5, p.933-944, 2004.

Recebido para publicação em 19 de março de 2004. Aceito para publicação em 25 de maio de 2004. 